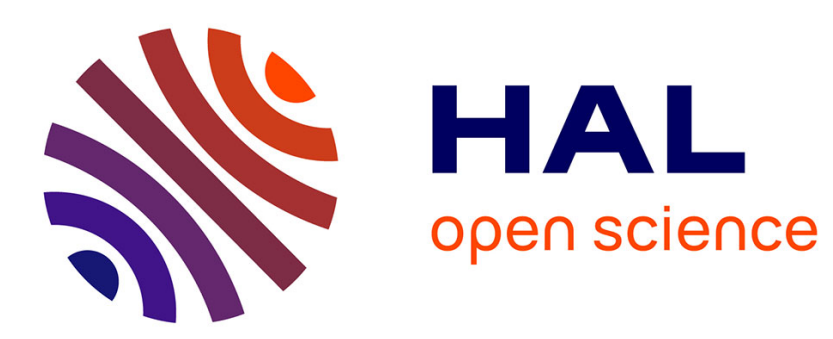

\title{
Du technicien au gestionnaire: regard sur les mutations de la filière sportive territoriale
}

Denis Bernardeau Moreau, Fabrice Bolot

\section{To cite this version:}

Denis Bernardeau Moreau, Fabrice Bolot. Du technicien au gestionnaire: regard sur les mutations de la filière sportive territoriale. Revue française d'administration publique, 2009, 131, pp.583-594. hal-00827898

\section{HAL Id: hal-00827898 \\ https://hal.science/hal-00827898}

Submitted on 2 Jun 2013

HAL is a multi-disciplinary open access archive for the deposit and dissemination of scientific research documents, whether they are published or not. The documents may come from teaching and research institutions in France or abroad, or from public or private research centers.
L'archive ouverte pluridisciplinaire HAL, est destinée au dépôt et à la diffusion de documents scientifiques de niveau recherche, publiés ou non, émanant des établissements d'enseignement et de recherche français ou étrangers, des laboratoires publics ou privés. 


\title{
DU TECHNICIEN AU GESTIONNAIRE : REGARD SUR LES MUTATIONS DE LA FILIERE SPORTIVE TERRITORIALE
}

\author{
Denis Bernardeau Moreau (sociologue et ancien directeur territorial) et Fabrice Bolot (juriste \\ et ancien formateur CNFPT en charge du domaine sport)
}

Tous les travaux s'intéressant au monde territorial témoignent d'une mutation profonde dans la façon de penser le territoire aujourd'hui. Ces évolutions de fond, voulues par le législateur, ont des incidences profondes sur les acteurs des institutions locales qui conduisent et accompagnent le changement. De plus en plus diplômés et gestionnaires «dans l'âme », ces acteurs sont au cœur des problématiques liées aux formes nouvelles de gouvernance locale ${ }^{1}$. L'histoire de la fonction publique territoriale est ancienne. Les premiers employés de ville remontent au XIIè siècle et s'occupent des voiries, des fontaines ou des actes administratifs ${ }^{2}$. Lors de la création des conseils municipaux en 1789, ils sont placés sous l'autorité des maires. Ces premières tentatives d'autonomie locale trouvent un nouvel élan avec la IIIe République et les premières lois de décentralisation de 1882 (relative aux départements) et de 1884 (relative aux communes). Si les bases d'une reconnaissance des agents territoriaux sont alors posées, c'est en 1952 qu'une loi (texte du 28 avril relatif au statut des fonctionnaires communaux) institue réellement le statut du personnel communal et hiérarchise les emplois en catégories. Puis, durant les décennies qui suivent, la situation évolue peu. Il faut attendre la mise en œuvre des grandes lois de la décentralisation des années 1982-83 (acte I de la décentralisation $^{3}$ ) pour que la situation se modifie fortement. Dès lors, la fonction publique territoriale (FPT) s'agrandit et va comprendre trois catégories de fonctionnaires à l'image de ceux de l'ÉTAT. Un nouveau cadre législatif fonde les bases actuelles du statut général de la fonction publique et organise les dispositions statutaires ${ }^{4}$. L'extension de la « clause générale de compétence » permet dès lors à chaque collectivité de décider librement de l'organisation de ses services publics territoriaux selon les priorités fixées par ses instances délibératives (conseil régional pour la région, conseil général pour le département et conseil municipal pour la commune). Il en va de même pour le recrutement des personnels, notamment des cadres. Avec le concours du tissu associatif durablement enraciné, la vie locale va progressivement trouver une véritable impulsion politique mais aussi professionnelle grâce à une fonction publique territoriale dynamique et fortement structurée comptabilisant aujourd'hui 1,7 million d'agents répartis dans huit filières et quelque 250 métiers différents répertoriés sur l'ensemble du territoire ${ }^{5}$.

\footnotetext{
${ }^{1}$ Voir par exemple Le Galès (Patrick) et Lorrain (Dominique), « Gouverner les très grandes métropoles », Revue française d'administration publique, 2003/3, n $107, \mathrm{p} .305-317$. Ces auteurs montrent que l'émergence des «mégamétropoles » pose, aux acteurs de terrain, des problèmes de gouvernabilité et favorise la construction d'institutions locales spécifiques au contexte local.

${ }^{2}$ Comme le souligne Durand (Dominique) Une histoire de la fonction publique territoriale, Paris, La dispute, Essai, 2004), les emplois de ville sont alors très divers : on compte par exemple des écuyers, des serruriers, des sonneurs de cloche, des maîtres d'école, des secrétaires-greffiers, des commis aux écritures, des garçons de bureau, des croque-morts ou des concierges.

${ }^{3}$ Il s'agit notamment de la loi du 02 mars 1982 relative aux droits et libertés des communes, départements et régions (loi sur l'organisation), de la loi du 17 juillet 1982 portant réforme de la planification, de la loi du 31 décembre 1982 définissant les statuts particuliers de Paris, Lyon et Marseille (loi PLM), des lois du 07 janvier 1983 et du 22 juillet 1983 relatives à la répartition des compétences (lois sur les compétences) et des lois du 13 juillet 1983 et du 26 janvier 1984 créant la fonction publique territoriale (droits et devoirs des fonctionnaires).

${ }^{4}$ Il s'agit de la loi du 13 juillet 1983 portant droits et obligations des fonctionnaires, de la loi du 26 janvier 1984 portant dispositions statutaires relatives à la fonction publique territoriale et de la loi du 12 juillet 1984 relative à la formation des agents de la FPT.

${ }^{5}$ Les filières sont les suivantes : administrative, technique, médico-sociale, culturelle, sportive, animation, police municipale, pompiers professionnels. Les filières administrative et technique sont les premières à être créées,
} 
La conduite du changement à l'échelle territoriale touche aujourd'hui l'ensemble des filières de la fonction publique. Si, dès les années 1950, des auteurs s'intéressent à l'administration territoriale $^{6}$, celle-ci est décrite comme une bureaucratie locale constituant bien souvent une petite société déjà très hiérarchisée ${ }^{7}$. Avec les grands mouvements de décentralisation, les politiques publiques se développent. Progressivement, elles s'étoffent et diversifient leurs approches $^{8}$. Avec la décentralisation, les professions territoriales se structurent aussi. Si certains auteurs dessinent le cadre général de la redéfinition des compétences ${ }^{9}$, d'autres vont opter pour une analyse plus sectorisée ; c'est le cas de Saunier et Claude ${ }^{10}$ qui s'intéressent à la constitution des savoirs chez les architectes urbanistes. C'est le cas également de Dupuy et Thoning ${ }^{11}$ à propos des administrateurs locaux, de Faget et $a l^{12}$ concernant les agents locaux de médiation sociale ou encore plus récemment de $\mathrm{Bah}^{13}$ étudiant les modes de recrutement des agents territoriaux des écoles maternelles. Dans le champ du sport, des travaux récents décrivent les modalités d'organisation et d'accompagnement du changement vers une forme plus gestionnaire des services municipaux ${ }^{14}$. Peu à peu, les politiques sportives des collectivités sont mises en œuvre dans un contexte juridique plus complexe. De nombreux textes sont publiés depuis les années 1990 sur la sécurité des équipements, sur l'organisation des manifestations sportives, sur les conditions d'encadrement des APS. L'offre sportive devient aussi plus individualisée et modulable pour répondre aux demandes multiples des différents publics. Dans un espace politique où les problématiques liées au territoire trouvent une résonance accrue, nous pouvons supposer que les compétences des agents territoriaux des sports tendent à fortement évoluer. Cette évolution est la conséquence de la décentralisation orchestrée maintenant depuis trois décennies et qui a conduit à une réelle reconnaissance statutaire de la fonction publique territoriale. Elle est aussi une réponse aux problématiques de performance qui touchent l'ensemble des collectivités. La professionnalisation des agents territoriaux est à l'oeuvre comme en témoignent les récentes modifications législatives qui

suivies de manière étalée par les autres filières. Selon le rapport annuel du ministère de la fonction publique de 2006, $69 \%$ des agents travaillent dans les communes, $11 \%$ dans les structures intercommunales, $18 \%$ dans les départements et $0,8 \%$ dans les régions. On compte près de $70 \%$ d'agents titulaires (Guide pratique de la fonction publique territoriale du CNFPT 2007).

${ }^{6}$ Crozier (Michel), « Pour une sociologie de l'administration publique », Revue française de science politique, Volume 6, n4, 1956, p.750-769.

7 Rocher (Guy), «Le sociologue et la sociologie dans l'administration publique et l'exercice du pouvoir politique », Sociologie et Sociétés, volume 12, n², octobre 1980, p.45-65.

Voir De Maillard (Jacques) et Kubler (Daniel), Analyser les politiques publiques, Voiron, Presses Universitaires Grenoble, 2009. Voir aussi Chaudoir (Philippe) et De Maillard (Jacques), (dir.), Culture et politique de la ville, Editions de l'Aube, Collection Mondes en cours, 2004. Ces auteurs soulignent par exemple l'intérêt porté aujourd'hui aux actions culturelles et sociales pour définir et développer les politiques de la ville.

${ }^{9}$ Voir par exemple Brisson (Jean-François), Les transferts de compétences de l'Etat aux collectivités locales, L'harmattan, Droit des Collectivités Territoriales, 2009.

${ }^{10}$ Saunier (Pierre-Yves) et Claude (Viviane), « Compétences urbanistes, compétences urbaines : état des lieux 1900-1930 », Vingtième siècle, novembre-décembre 1999. Sur la structuration des urbanistes de profession, voir aussi Le Saunier (Pierre-Yves), «Où l'auteur propose un plan de travail pour une histoire dite sociale de l'aménagement urbain », Recherche contemporaine, $\mathrm{n}^{\circ} 3$, 1995-1996.

11 Dupuy (François) et Thoenig (Jean-Claude), Réformer ou déformer, la formation permanente des administrateurs locaux, Paris, Cujas, 1980.

12 Faget (Jacques); Maillard (Jacques de), Sene Diop (Moussa) et Caquant (Anne), Les agents locaux de médiation sociale en quête d'identité, Paris, Editions de l'IHESI, Etudes et recherches, 2003.

${ }^{13}$ Bah (Mamadou) (sous la direction de Saglio Jean), Thèse intitulée « Entre recrutement local des femmes de service et statut national des ATSEM. Analyse sociologique de deux modes de gestion des marchés du travail dans la FPT », soutenue le 21 novembre 2008 à l'UPMF de Grenoble.

${ }^{14}$ Chaze (Jean-François), «Les évolutions du management municipal, Le développement du sport », Corps et culture, $\mathrm{n}^{\circ} 1,1995$. Gasparini (William) et Scheeck (Luc), «Organiser le changement dans un service municipal des sports ", Revue européenne de management du sport, $\mathrm{n}^{\circ} 2$, 1999. Noir (Christian), «Politique sportive et management du sport » in Loret (Alain), Sport et management, Paris, Dunod, 1993, p.90-102. 
entendent spécialiser le processus de formation des fonctionnaires territoriaux ${ }^{15}$, comme en témoignent également les nombreuses informations collectées auprès des acteurs territoriaux. Ces derniers sont nombreux aujourd'hui à réclamer une reconnaissance de leurs métiers, une meilleure adéquation de leurs formations initiales et continues ${ }^{16}$ et l'acquisition de compétences plus gestionnaires. Affichant leur appartenance à un corps professionnel dont le niveau d'accès est désormais identique à la fonction publique d'ÉTAT, une partie des agents territoriaux se revendique désormais comme des gestionnaires confrontés aux problématiques économiques (maîtrise des coûts), concurrentielles (la multiplication des cas de délégation de service public au secteur commercial atteste que le service public n'est plus la prérogative exclusive des agents publics) et clientélistes (car le public agit aussi comme un client prêt à répondre au plus offrant). L'évolution du processus de professionnalisation dans la fonction publique territoriale conduit ainsi les agents territoriaux des sports à l'adaptation de leurs compétences et à la rationalisation de leur fonctionnement en réponse à des situations plus managériales. Ce sont des profils d'agents différents qui se dessinent. Certaines études montrent que ces différences sont générationnelles (il y a les anciens et les nouveaux), nous verrons que notre analyse différencie deux groupes d'agents en fonction des formations sportives et scolaires qu'ils ont suivies: Il y a les techniciens sportifs d'un coté et les gestionnaires du sport de l'autre. Entre ces deux catégories d'agents se distinguent deux manières de vivre son activité professionnelle au service du territoire.

Pour illustrer nos propos, notre étude s'appuie sur une quarantaine d'entretiens effectués avec des acteurs locaux issus de la filière sportive. Des agents de communes et de départements (directeurs et éducateurs territoriaux), de la ville de Paris (dont les statuts territoriaux sont particuliers) et du Centre national de la fonction publique territoriale (CNFPT), ont été interrogés entre février 2008 et janvier 2009. L'échantillon a été constitué par réseau jusqu'à atteindre son point de saturation, au-delà duquel aucune information nouvelle n'est recueillie. Notre article comprend deux parties traitant dans un premier temps de l'orientation gestionnaire des services territoriaux des sports, présentant une spécialisation des tâches des agents territoriaux et une formalisation accrue. Puis, dans un second temps, nous analyserons comment les missions managériales sont assumées par ces agents dont la représentation du métier s'est, pour certains, fortement transformée.

\section{Le service des sports, une gestion au service du territoire}

\section{I.1. La naissance de la filière sportive territoriale}

Le développement du sport en FRANCE remonte à la fin du XIX ${ }^{\circ}$ siècle sous l'impulsion des pouvoirs publics et des fédérations sportives. A l'échelle locale, les principaux acteurs sont les associations, les élus politiques chargés des sports, mais aussi les salariés fonctionnaires et contractuels du champ sportif territorial. Au cours des dernières décennies, l'importance du sport va croître considérablement jusqu'à figurer, désormais, dans la plupart des politiques

\footnotetext{
${ }^{15}$ La loi du 19 février 2007 relative à la fonction publique territoriale (FPT) a réorganisé les formations des agents territoriaux et opéré une nouvelle répartition dans l'organisation des concours, entre les Centres de gestion (concours A, B, C) et le CNFPT qui prend en charge les concours des cadres classés en catégorie «A+ » (les administrateurs territoriaux notamment).

${ }^{16}$ Aujourd'hui, il n'y a plus à proprement parler de formation initiale (formation avant titularisation) et continue (formation d'adaptation à l'emploi). Les formations obligatoires comprennent depuis la loi du 19 février 2007 des actions d'intégration (dispensées en début de carrière aux agents des trois catégories) et de professionnalisation (dispensées tout au long de la carrière et à l'occasion de l'affectation dans un poste de responsabilité). Ces dispositifs - dont les programmes seront mis en œuvre par le CNFPT - sont ainsi liés à la vie statutaire mais aussi à l'évolution de la situation professionnelle.
} 
territoriales ${ }^{17}$. A l'origine, l'histoire du sport territorial est étroitement liée à la construction des premiers équipements, notamment pour les scolaires ${ }^{18}$. Pour Haschar-Noé, c'est une étape décisive dans la structuration des services des sports ${ }^{19}$. Avec la construction massive des piscines notamment, dans les années 1970, pour résorber le risque de noyade d'enfants sur les bords de mer, les collectivités doivent recruter du personnel spécialisé, les maîtres nageurs sauveteurs (MNS), dont la profession est régulée dès 1951 (loi du 24 mai). Ces premiers recrutements expliquent aussi pourquoi les directeurs de service des sports sont très souvent d'anciens spécialistes de la natation. Puis, pour répondre à la demande des instituteurs et des parents, va s'ajouter progressivement une autre catégorie d'agent territorial, plus polyvalente et plus mobile aussi : les moniteurs municipaux des sports ${ }^{20}$. Tandis que les maîtres nageurs, recrutés pour surveiller les bassins, voient leurs tâches s'élargir à l'entraînement de la natation puis à l'enseignement d'activités aquatiques diversifiées (bébés nageurs, aquagym, etc.), les moniteurs sont affectés à l'enseignement de l'éducation physique et sportive auprès des écoles primaires puis au sein des écoles municipales des sports. Le patrimoine sportif se diversifiant considérablement, la spécialisation des agents se systématisant, la mise en place des services des sports autonomes se généralise au début des années 1980. Dès lors, la question de la place et de la légitimité de ces services va permettre de juger de l'importance accordée aux sports et aux moyens de leur mise en œuvre par des professionnels issus du milieu, mais dont les statuts, au début des années 1990, restent encore à inventer. Devant l'enjeu croissant des politiques sportives territoriales, la nécessité criante de donner un statut adapté au fonctionnaire sportif territorial et de renforcer sa légitimité professionnelle devient l'objet de revendications répétées, émanant notamment du syndicat national des directeurs des sports (SNDS). Ces revendications vont conduire le législateur à créer, en 1992, la filière sportive territoriale. Celle-ci remplace l'ancien statut communal dont les caractéristiques étaient l'absence de catégorie A et un recrutement sur titre ${ }^{21}$. Les décrets organisent trois cadres d'emplois sportifs au sein des collectivités. Il s'agit des conseillers territoriaux des activités physiques et sportives (CTAPS) de catégorie A, des éducateurs territoriaux des activités physiques et sportives (ÉTAPS) de catégorie B et des opérateurs territoriaux des activités physiques et sportives (OTAPS) de catégorie C. Progressivement, les agents des services municipaux des sports se voient investis de nouvelles missions concernant la gestion du sport et doivent redéfinir leurs secteurs d'intervention (gestion des équipements de proximité, management des personnels d'encadrement, rationalisation des coûts de fonctionnement ...).

\footnotetext{
${ }^{17}$ Bayeux (Patrick) et Dupuis (Jérôme), Les politiques publiques sportives territoriales. De l'élaboration à l'évaluation, Paris, Revue EPS, Sport et collectivités territoriales, 1999. Chaboche (José), «Les stratégies des Conseils généraux dans le développement sportif territorial », Revue européenne du sport, $\mathrm{n}^{\circ} 5,2001$. HascharNoé (Nadine), «L'évolution des politiques sportives municipales en France : entre rationalisation et construction identitaire », Revue Loisir et Société/Society and Leisure, volume 27, n², 2004, p 489-527. Monneret (Jean), Les politiques sportives des collectivités territoriales, Paris, Vigot, Sport et enseignement, 1998. Quantin (Jacques), Missions et organisation du service territorial des sports, Paris, EPS CNFPT, 1993.

${ }^{18}$ Bonnenfant (Roger), «Les équipements sportifs », Revue Française de Marketing, 3, 1992, p.61-80. Callède (Jean-Paul), «Le processus de développement des équipements sportifs et culturels », Revue économique du Sud Ouest, ${ }^{\circ} 1$, 1988, p.41-66. Falcoz (Marc) et Chifflet (Pierre), «La construction publique des équipements sportifs », Les annales de la recherche urbaine, Sports en ville, n79, 1998, p.14-21.

19 Haschar-Noe (Nadine), «Les politiques sportives locales et leur management en France : état des connaissances et des recherches », in Bouchet (Patrick) et Pigeassou (Charles) (dir.), Management du sport : Actualités, développements et orientations de la recherche, Afraps, 2006, p.242.

${ }^{20}$ Les statuts du personnel des sports prévoient à l'époque trois cadres d'agents : les aide-moniteurs de catégorie $\mathrm{C}$, les moniteurs de sport et les maîtres nageurs sauveteurs, tous deux de catégorie B.

${ }^{21}$ Les emplois d'aide-moniteur, de moniteur et de maître nageur sauveteur étaient alors accessibles par concours, tandis que les emplois de moniteur-chef, de chef de bassin et de chef de service des sports n'étaient accessibles que par avancement.
} 


\section{I.2. Des services des sports autonomes et gestionnaires}

« Nous sommes là pour gérer l'économie d'un service »(Jean-Luc).

Les premières études attestant de l'orientation gestionnaire des services des sports datent des années 1990. Noir par exemple ${ }^{22}$ montre comment, face à l'autonomie et à la gestion plus économique du sport local, certains services municipaux se sont structurés, ont rationalisé leurs processus de décision et optimisé leur mode de fonctionnement. $\mathrm{Chaze}^{23}$ décrit ce processus de transformation des services municipaux des sports, depuis leur création jusqu'à leur forme actuelle de plus en plus managériale. L'auteur conclut que l'organisation du service municipal des sports répond de plus en plus désormais à un «management stratégique ». L'augmentation des dépenses consacrées au sport (selon l'INSEE, les dépenses des collectivités locales consacrées au sport sont passées de 1,2 milliards d'euros en 1980 à 9,6 milliards d'euros en 2006, situant actuellement le budget sport à environ $6 \%$ du budget global), comme le recrutement constant d'agents de la filière sportive (selon le CNFPT, on compte 12700 agents en 1996, 16400 en 2003 et plus de 20000 aujourd'hui) témoignent de l'intérêt des élus pour ce secteur. Peu à peu, l'intervention sportive locale devient indispensable. Selon diverses enquêtes du $\mathrm{CNFPT}^{24}$, les services des sports existent désormais dans plus de $70 \%$ des communes de plus de 5000 habitants (mais l'absence d'un service des sports ne signifie pas pour autant l'absence de politique sportive car la plupart des collectivités locales appliquent une politique sportive, même embryonnaire, ne serait-ce que par le versement de subventions aux associations ${ }^{25}$ ). Ces services communaux ne sont pas tous rangés au même niveau. Parfois peu considérés, ceux-ci voient alors leurs tâches cantonnées à quelques secteurs d'intervention épars et limités. Le plus souvent, ils restent rattachés à un service technique ou administratif, technique parce que les collectivités sont propriétaires d'installations sportives qui demandent de l'entretien confié aux agents techniques, et administratif parce que le service des sports prenant de l'ampleur doit recruter du personnel administratif spécifique ${ }^{26}$. Quand l'autonomie du service des sports vient enfin à se réaliser, elle exprime très souvent une volonté des élus voulant organiser une véritable politique sportive. Identifiant trois étapes de construction des politiques sportives, Noé ${ }^{27}$ constate que la période actuelle dite de rationalisation (les deux précédentes correspondant à l'émergence puis la normalisation des politiques sportives) indique une plus forte responsabilisation des élus. Cette période s'accompagne du recrutement de personnels administratifs mieux formés à la gestion des services territoriaux.

\section{I.3. Vers une spécialisation des agents territoriaux}

\footnotetext{
${ }^{22}$ Noir (Christian), op. cit.

${ }^{23}$ Chaze (Jean-François), op. cit.

${ }^{24}$ Le CNFPT réalise et commande régulièrement des enquêtes sur les agents et les métiers territoriaux. Voir notamment les enquêtes du CNFPT : «Les services des sports des villes de plus de 5000 habitants » (1990) et «Les politiques sportives des villes » (1992) accessibles sur le site de l'institution.

${ }^{25}$ Bolot (Fabrice), «Les aides apportées par les collectivités territoriales aux clubs sportifs : le cas de l'aide aux clubs de football », La gazette du Palais, n $^{\circ} 37-39,2005$, p. 6-11.

${ }^{26}$ Il est d'ailleurs fréquent, encore aujourd'hui, de constater des conflits de compétences entre le service des sports et le service technique, dans la mesure où les installations sportives réclament à la fois un entretien des locaux (à la charge le plus souvent du service des sports, sauf il est vrai les gros travaux) et des espaces verts (où on ne sait trop qui, du service technique ou du service des sports, en a la charge).

${ }^{27}$ Noé (Nadine), Contribution à l'analyse socio-historique des politiques sportives municipales : émergence, transformation et différenciation. Le cas du Val d'Yerres en Région parisienne (1965-1995), Université de Paris XI, 1995 (non publié).
} 
Toute organisation du travail inclut une division verticale et horizontale du travail par la division des tâches, la formalisation des liens hiérarchiques et des modalités de communication et de coordination. Pour juger de l'évolution d'une organisation, Hatch ${ }^{28}$ propose de considérer plusieurs «dimensions structurelles» dont la spécialisation, la formalisation et la centralisation. La spécialisation des tâches peut être mesurée verticalement par le nombre de niveaux hiérarchiques du plus élevé au plus bas. Dans les collectivités territoriales, elle s'observe aussi à travers les catégories d'emplois correspondant à des niveaux de responsabilités. Parmi les tendances relatives aux transformations du phénomène local, notent Callède et Dané, figure «une tendance à la spécialisation des tâches et des statuts $»^{29}$. D'après le Guide pratique de la fonction publique territoriale de 2007 , pour $9 \%$ d'agents et de cadres de catégorie A, on compte $14 \%$ d'agents de catégorie B et $77 \%$ d'agents de catégorie C. Dans nos études de cas, la répartition est de $8 \%$ de cadres A, 30\% d'agents de catégorie B (du fait notamment d'un nombre élevé d'éducateurs sportifs) et $62 \%$ d'agents de catégorie $\mathrm{C}$ (agents techniques et d'entretien surtout, gardiens et personnel d'accueil). Dans cette pyramide des niveaux hiérarchiques, l'encadrement des agents (notamment ceux opérant sur le terrain) est une contrainte évidente corroborant les propos de nos cadres interviewés expliquant que $80 \%$ de leur temps est consacré à la gestion de leur personnel. Hier éducateurs ou surveillants, les agents des services des sports voient la gamme de leurs missions considérablement s'allonger et se spécialiser. Ils sont animateurs et éducateurs sportifs mais aussi « responsable des relations avec les comités départementaux» (Hadi), « responsable de projet» (Jean-Louis), «responsable des activités sportives» (Michel), «responsable des installations » (Patrick), «manager du service des sports » (Franck) ou «manager d'équipe » (Fred). La formalisation renseigne sur la spécialisation horizontale des fonctions et modes de coordination entre les acteurs. Plus les procédures et les organigrammes sont importants, note Hatch, et plus la formalisation est élevée. On parle de structure divisionnelle (ou « en râteau ») quand la répartition des agents se fait par secteur d'installation. Cette forme d'organisation est la plus traditionnelle. On parle de structure fonctionnelle quand la répartition des agents se fait davantage par fonction. Les structures territoriales que nous avons étudiées sont divisionnelles quand il s'agit de petites villes (Collégien, Garges-lèsGonesse, Roissy-en-Brie), identifiant des lieux géographiques (gymnases, accueil, piscines, stades). Elles sont fonctionnelles quand la ville est de moyenne ou grande importance (SAN de Sénart, SAN du Val d'Europe, Dunkerque, Meaux), distribuant les agents selon les fonctions qu'ils remplissent (direction, animation, entretien des équipements, soutien aux associations, organisation des évènements). Parfois aussi, la structure présente un mixte des deux (Besançon par exemple). Quant à la centralisation, elle est forte si les décisions sont prises au sommet de l'organisation. Le mode de gouvernance observé dans les collectivités territoriales de notre échantillon montre majoritairement une forte centralisation des décisions - les élus restent les seuls décideurs en matière politique - mais les agents territoriaux ont un rôle de conseil aux élus et d'expertise indiscutable. Dans ce «noyau stratégique » ${ }^{30}$ dominé par l'élu, les décisions opérationnelles et administratives sont fortement décentralisées et relèvent de la compétence des agents. Dans ce mode de direction où l'élu reste la pièce maîtresse siégeant au conseil exécutif, il apparaît que la gouvernance privilégie en définitive le mode participatif en institutionnalisant une répartition équilibrée des pouvoirs politiques au sommet et gestionnaires le long de la chaîne administrative.

\footnotetext{
${ }^{28}$ Hatch (Mary Jo), Théorie des organisations - de l'intérêt de perspectives multiples, Bruxelles, De Boeck, 2005.

${ }^{29}$ Callède (Jean-Paul) et Dané (Michel), Sociologie des politiques sportives locales. Trente ans d'action sportive à Bègles 1959-1989, Institutions sportives et sociétés locales, Talence, MSHA, 1991, p.184.

${ }^{30}$ Mayaux (François), Noyau stratégique des associations : quel partage des pouvoirs entre dirigeants bénévoles et dirigeants salariés? Thèse de Doctorat. Sciences de Gestion. Lyon 3, 1996.
} 


\title{
II. Du sportif gestionnaire au gestionnaire du sport
}

\author{
«J'étais un éducateur de terrain. Aujourd'hui, je me considère comme un sportif \\ bureaucrate » (Philippe $\mathrm{P})$.
}

\section{II.1. Deux visions du métier territorial des sports}

Les cadres d'emplois de la filière sportive précisent les conditions d'exercice des agents territoriaux : Les CTAPS sont les directeurs de service des sports et ont la responsabilité de l'ensemble des activités et de leur personnel d'encadrement conformément aux orientations définies par l'autorité exécutive territoriale. Les ÉTAPS conduisent sur le plan administratif, social, technique, pédagogique et éducatif, les activités physiques et sportives de la collectivité. Ils assurent la sécurité du public et des installations. Dans le statut des ÉTAPS sont inclus les éducateurs sportifs en charge de l'enseignement de l'EPS, mais aussi les maîtres nageurs sauveteurs. Quant aux OTAPS, ils sont chargés d'assister en règle générale les éducateurs dans leurs tâches de surveillance des piscines et des baignades.

A l'époque, la création de la filière sportive répond à deux attentes fortes. Celle des élus s'inscrit dans un contexte de développement des enjeux du sport territorial suite à une décentralisation très peu explicite dans ce domaine. En même temps qu'ils prennent à leur charge l'organisation du sport local, les élus intègrent sa réelle dimension politique et sociale. Ils prennent alors conscience que la direction d'un service des sports et la mise en place d'une réelle politique sportive ne sont pas toujours conciliables avec les profils de directeurs dont les compétences sont essentiellement sportives par leurs expériences d'anciens professeurs de sport, d'entraîneurs ou de maîtres nageurs sauveteurs. Apprenant leur métier d'élu local qui, souligne Savary ${ }^{31}$, se complexifie lui aussi et nécessite des compétences accrues et de plus en plus pointues, il s'agit pour eux de développer leurs politiques sportives avec l'aide de cadres formés à la gestion, au management et au droit, aptes à mobiliser des compétences transversales. A cette attente en répond une autre : celle des agents eux-mêmes qui veulent obtenir une « réelle reconnaissance statutaire » (Jean-Louis) permettant de «hiérarchiser les professions et les compétences correspondantes »(Michel). Cette reconnaissance concourt à la construction de l'identité professionnelle permettant à l'individu de se situer dans un groupe de travail. Pour les acteurs locaux du sport, «il y a depuis longtemps une véritable envie de se faire reconnaître avec les spécificités qui existent dans ces carrières propres aux collectivités territoriales »(Christophe $\mathrm{N}$ ). Si une enquête de l'Association nationale des directeurs et intervenants d'installations et des services des sports (ANDISS) ${ }^{32}$ montre que la réussite au concours de CTAPS est grandement facilitée par la connaissance du métier (la plupart des lauréats interrogés ont, au moment du concours, une expérience déjà significative dans des services des sports), une autre enquête réalisée par la faculté des sciences du sport de Marseille $^{33}$ conclut que le CTAPS présente deux profils : Soit il est un technicien issu du sérail, enraciné dans sa commune comme ancien sportif, qui a évolué par promotion ou concours interne. Soit il est un manager territorial, plutôt jeune et diplômé de niveau bac +3 et plus, mobile, ambitieux mais pas nécessairement sportif. L'enquête souligne également que pour les conseillers interrogés, les compétences requises par leur métier sont de plus en plus le management des ressources humaines, le conseil aux élus et aux associations et l'aspect gestionnaire et administratif. Travailler dans un service des sports est «un véritable métier

\footnotetext{
${ }^{31}$ Savary (Emile), Le «métier » d'élu local. Repères pour se situer et agir, Lyon, Chronique sociale, 2007.

${ }^{32}$ Enquête de Poitou (Bernard), 2003 (accessible sur le site aires.asso.fr).

${ }^{33}$ Bluteau (Serge), Etude en PACA, faculté des sciences du sport, Marseille, 2005 (accessible sur le site de l'ANDISS).
} 
parce qu'on n'est pas dans un seul domaine. Le domaine est technique, mais aussi physiologique, humain ou encore mental. Tout est lié, on est dans l'organisation et la planification (...), ça demande des compétences transversales »(Emmanuel). Alors que certains dirigeants complètent leurs expériences professionnelles et sportives par des formations en management et en gestion, de nouveaux cadres issus des filières universitaires viennent progressivement remplacer les anciens techniciens du sport.

L'analyse de notre échantillon confirme l'existence de ces deux profils d'agents territoriaux des sports soulignés par les études précédemment citées. La différence essentielle que nous relevons n'est pas tant l'âge mais davantage la formation suivie et le type de diplôme validé. Le groupe ayant un profil que nous qualifions de technique est plutôt jeune ( 25 ans), de niveau Bac ( $\mathrm{S}$ de préférence), titulaire du brevet d'ÉTAT, ayant un bon niveau sportif et occupant un poste de maître nageur sauveteur ou d'éducateur territorial. Le groupe que nous qualifions de gestionnaire est plus âgé (40 ans), nettement plus diplômé (de niveau Bac +4 plutôt dans le management, le marketing ou le droit du sport), titulaire d'un brevet d'ÉTAT (mais en proportion moindre), ayant un niveau sportif moyen et occupant un poste d'éducateur ou de conseiller. Entre ces deux profils, ce sont les discours, les positionnements et les représentations du métier qui se distinguent et peuvent s'expliquer par la nature des formations dispensées. Techniques quand il s'agit du brevet d'ÉTAT, plus globales quand il s'agit des cursus universitaires, les formations soulignent ainsi deux perceptions du monde sportif. Celle du ministère des sports (lui seul organise les brevets d'ÉTAT) tend à former des éducateurs et des entraîneurs immédiatement opérationnels dans un rapport qualifié $\mathrm{d}^{\prime}$ homme/technique ${ }^{34}$. En revanche, la vision du ministère de l'ÉDUCATION NATIONALE se veut plus générale et positionnée sur un rapport homme/environnement ${ }^{35}$. Entre les deux ministères, la concurrence existe, accentuée par la reconnaissance professionnelle des diplômes universitaires inscrits désormais au Registre national des certifications professionnelles $\left(\mathrm{RNCP}^{36}\right)$. Le milieu social tend à façonner l'individu à son image. Il en est de même pour la formation qui légitime un modèle social allant de soi pour l'élève. Tandis que les techniciens sportifs déclarent mobiliser des compétences pédagogiques, relationnelles et sécuritaires, les gestionnaires du sport déclarent utiliser des compétences managériales et transversales. La réalité des premiers traduit une vision très technicienne de leur métier et de son utilité - «Un bon éducateur est quelqu'un qui doit amener une personne vers de la performance » (Matthieu). En revanche, les seconds lisent leur métier à travers le prisme du gestionnaire mobilisant des ressources et des projets. "Je suis un "gestionnaire» des demandes des écoles primaires et un « manager » des animateurs sportifs au sein du service »

\footnotetext{
${ }^{34}$ Chifflet (Pierre), «Savoirs sportifs et formation des cadres » in Bruand (Gérard) (coord.), Savoirs et sens pratique dans les APS, Acte du symposium organisé par l'AFRAPS le 23 octobre, 1988, p.137-148.

${ }^{35}$ Chifflet se réfère aux travaux de Colardyn (Danielle) et Lantier (Françoise), «L'analyse des contextes professionnels : quelles problématiques pour quels objectifs de formation ? », Revue Française de Pédagogie, Paris, $\left.n^{\circ} 61,1982\right)$. Ces auteurs identifient trois générations de problématiques selon les types de relation possibles entre l'homme et les systèmes de travail. La première génération établit un rapport homme/technique où l'apprentissage a pour objectif d'apporter des savoirs internes, des connaissances fermées aux métiers immédiatement transférables à l'activité professionnelle. La deuxième génération établit un rapport homme/organisation dans lequel la formation doit permettre à l'apprenant de favoriser une mobilité professionnelle au sein de l'organisation afin d'adapter le métier aux évolutions de l'organisation. Enfin, la troisième génération de problématique est la plus ambitieuse car dans le rapport homme/environnement, elle prend en compte les transformations de l'environnement pour développer des compétences transversales et transférables à différentes situations nouvelles.

${ }^{36}$ Ce répertoire, à disposition de l'ensemble des professionnels (employeurs et employés), tient à jour les informations relatives aux diplômes et titres à finalité professionnelle ainsi que les certificats de qualification. Sont mentionnés les activités visées, le secteur d'activité, les compétences acquises, les modalités d'accès, les niveaux de responsabilité, tels que définis par les commissions paritaires nationales de l'emploi des branches professionnelles.
} 
(Gérard). «Aujourd'hui, nous dit un directeur de service, on a d'un côté des agents issus des filières techniques, des professeurs de sport ou des maîtres nageurs qui sont d'une autre école et qui font au mieux, et de l'autre coté, on a des managers qui sont très bien formés et très compétents ». Pour ces derniers, l'évolution de la filière sportive va dans le sens d'une plus grande dimension managériale dans les missions proposées. Le directeur des sports est aujourd'hui "un véritable chef d'orchestre, écrit Monneret, tout autant technicien, gestionnaire, animateur que manager d'une équipe $»^{37}$. Les enjeux changent. La création de la filière et l'évolution de ses contenus de formation marquent la progressive incorporation des valeurs managériales par une partie des agents des services des sports.

\section{Profils types des personnes interviewées}

\begin{tabular}{|c|c|c|c|}
\hline & Représen & ion du métier & \multirow{4}{*}{$\begin{array}{l}\frac{\text { Caractéristique du groupe }}{\text { des techniciens sportifs }} \\
\text { plutôt jeune ( } 25 \text { ans), de } \\
\text { niveau Bac ( } \mathrm{S} \text { de } \\
\text { préférence), titulaire du } \\
\text { brevet d'Etat, de bon } \\
\text { niveau sportif et occupant } \\
\text { un poste de maître nageur } \\
\text { sauveteur ou d'éducateur }\end{array}$} \\
\hline \multirow{3}{*}{$\begin{array}{c}\text { Profil } \\
\text { technique et } \\
\text { sportif }\end{array}$} & $\begin{array}{l}\text { - Nous sommes des professionnels } \\
\text { de l'entraînement } \\
\text { - Nous sommes des professionnels } \\
\text { de l'enseignement } \\
\text { - C'est juste une activité rémunérée }\end{array}$ & $\begin{array}{l}\text { Arnaud, Richard, Said, Bruno, Yoann } \\
\text { Loïc, Henri, Lila, Jean, Mathieu } \\
\text { Loïc, Bruno, Sophie, Gérard }\end{array}$ & \\
\hline & \multicolumn{2}{|c|}{ Evolution des compétences } & \\
\hline & $\begin{array}{l}\text { - Compétences sécuritaires } \\
\text { - Compétences techniques et } \\
\text { d'encadrement } \\
\text { - Compétences pédagogiques et } \\
\text { relationnelles }\end{array}$ & $\begin{array}{c}\text { Mathieu, Loïc } \\
\text { Guéric, Sophie, Gérard, Jean, Richard, } \\
\text { Arnaud, Mathieu } \\
\text { Odile, Henri, Yoann, Sophie, Lila, Gérard, } \\
\text { Arnaud }\end{array}$ & \\
\hline \multirow{4}{*}{$\begin{array}{c}\text { Profil } \\
\text { managérial } \\
\text { et } \\
\text { gestionnaire }\end{array}$} & \multicolumn{2}{|c|}{ Représentation du métier } & \multirow{4}{*}{$\begin{array}{c}\text { Caractéristique du groupe } \\
\text { des gestionnaires du sport } \\
\text { Plutôt d'âge mûr (40 ans), } \\
\text { de niveau } \\
\text { Bac + } 4 \text { (plutôt en sport), } \\
\text { titulaire d'un brevet } \\
\text { d'Etat, de niveau sportif } \\
\text { moyen et occupant un } \\
\text { poste d'éducateur ou de } \\
\text { conseiller. }\end{array}$} \\
\hline & $\begin{array}{l}\text { - Nous sommes des managers } \\
\text { - Nous sommes des gestionnaires } \\
\text { - Nous sommes des mobilisateurs de } \\
\text { ressources } \\
\text { - Nous sommes des concepteurs de } \\
\text { projets }\end{array}$ & $\begin{array}{c}\text { Franck, Fred, Nathalie, Gérard C, } \\
\text { Christophe, Christophe J Jean-Louis } \\
\text { Gérard C, Jean-Louis, Bruno, Philippe P, } \\
\text { Emmanuel } \\
\text { Gérard, Jean-Louis } \\
\text { Fred, Gérard C }\end{array}$ & \\
\hline & \multicolumn{2}{|c|}{ Evolution des compétences } & \\
\hline & $\begin{array}{l}\text { - Compétences en culture générale et } \\
\text { ouverture d'esprit } \\
\text { - Compétences croisées et transversales } \\
\text { - Compétences pluridisciplinaires et } \\
\text { polyvalentes } \\
\text { - Compétences méthodologiques et en } \\
\text { conduite de projet } \\
\text { - Compétences en gestion des } \\
\text { ressources humaines }\end{array}$ & $\begin{array}{c}\text { Franck, Fred, Frédérick V, Nathalie } \\
\text { Fred, Nathalie, Hadi, Philippe P, Michel, } \\
\text { Farid, Patrick P, Emmanuel } \\
\text { Nathalie, Franck, Michel, Fred, Christophe, } \\
\text { Christophe J Frédéric V } \\
\text { Jean-Louis, Nathalie } \\
\text { Fred, Nathalie, Gérard C, Christophe, Jean- } \\
\text { Luc, Jean Nathalie, Christophe J, Philippe, } \\
\text { Frédéric V }\end{array}$ & \\
\hline
\end{tabular}

\section{II.2. Mieux gérer pour mieux travailler}

«Nous sommes des cadres et des managers, dotés de compétences accrues pour diriger nos équipes et nos structures. (...) Nous gérons, nous planifions, nous mettons en place les projets politiques de nos élus » (Christophe J).

\footnotetext{
${ }^{37}$ Monneret (Jean), op. cit., p. 52.
} 
Dans ce «mini-système social ${ }^{38}$, le métier territorial se caractérise par une «expertise affirmée » présente chez les cadres mais aussi chez les agents des catégories B et C. Les opérateurs comme les éducateurs ont eux aussi entamé leur «révolution» (expression employée par un de nos interviewés) et connaissent une mutation très forte dans la manière de concevoir leur métier. Même les gardiens de gymnases réclament plus de responsabilités en matière de sécurité et d'hygiène dans l'exercice de leur fonction et ne souhaitent plus restés cantonnés à une simple mission d'accueil et de surveillance. Les éducateurs demandent eux aussi plus de reconnaissance et d'autonomie dans la gestion de leurs activités, dans la proposition et la concrétisation des projets sportifs. Ils managent des scolaires, ils conseillent et «coachent» des associations. Les éducateurs affirment avec force qu'ils sont aussi des concepteurs de projets sportifs et des «animateurs managériaux ${ }^{39}$. «Il y a, reconnaît l'un d'eux, une grosse volonté des éducateurs d'évoluer vers du management de projet et de sortir de l'activité elle-même» (Christophe $\mathrm{N}$ ). Toutes ces mutations professionnelles sont clairement revendiquées par nos interviewés et sont autant de facettes de l'évolution des métiers de la filière sportive territoriale. Avec les lois de décentralisation, avec la nouvelle répartition des compétences, c'est autant d'agents territoriaux en plus pour les collectivités qui réclament une "forte dose de management» (Hadi). L'agent territorial est un expert, un « véritable coach» (Frédéric V.). Il réclame une meilleure reconnaissance de son travail, par ses élus et par les administrés aussi. Il veut montrer qu'il est capable de mettre en place des projets cohérents en s'appropriant les nouvelles orientations managériales qui, affirment nos interlocuteurs, sont symptomatiques de la modernisation en cours des services territoriaux des sports. Cette tendance est générale. Pour les élus, le fait de bénéficier des conseils de gestionnaires connaissant le milieu du sport et sensibilisés à sa culture est important. Ne serait-ce que pour coordonner l'action des associations sportives gourmandes d'installations et ayant tendance à s'en approprier l'espace (notamment dans les piscines où les clubs tendent à accaparer l'ensemble du bassin au bénéfice de leurs seuls nageurs). La connaissance de ces « coutumes » justifie que certains élus préfèrent s'entourer de gestionnaires issus du sérail sportif plutôt que d'administrateurs territoriaux des filières technique ou administrative. "Les élus se sont rendus compte des véritables enjeux de la pratique sportive et qu'à partir de là, on ne pouvait plus laisser le développement de la pratique sportive à des gens qui n'avaient aucune compétence sportive et managériale » (Franck). D'après une étude concernant une cinquantaine de collectivités ${ }^{40}$, une majorité d'entre elles $(70 \%)$ considèrent que leur politique s'inscrit de plus en plus dans une posture managériale, alliant démarches, outils et capacités d'anticipation et de synthèse (prospective territoriale, veille stratégique, prospective financière, systèmes de pilotage...), notamment en ce qui concerne la programmation des investissements et des projets à moyen et long terme. $75 \%$ des collectivités interrogées déclarent observer un développement du management de et par projet. Un tiers a recours à des tableaux de bord confirmant que l'utilisation des outils modernes de gestion se systématise et que «l'activité de tous s'inscrit dans une conception moderne du management des entreprises ${ }^{41}$. Parmi ces outils, certains sont destinés à réduire les coûts de fonctionnement des équipements sportifs (ratios par équipement, types d'usagers et taux de fréquentation), d'autres sont destinés à optimiser la gestion des ressources humaines (suivi des actions,

\footnotetext{
${ }^{38}$ Rocher (Guy), op. cit.

${ }^{39}$ Bolot (Fabrice), «Les éducateurs de la filière sportive territoriale ou la lente reconnaissance statutaire des « animateurs managériaux » du sport territorial », in Bernardeau Moreau (Denis) \& Collinet (Cécile) (dir.), Les éducateurs sportifs en France depuis 1945. Questions sur la professionnalisation, Rennes, PUR, novembre 2009, p.185-194.

${ }^{40}$ Enquête menée par Bernard Brunhes Consultants en partenariat avec l'Association des administrateurs territoriaux de France, «Vingt ans de décentralisation. Où en est aujourd'hui le management public territorial ?, Les cahiers, $\mathrm{n}^{\circ} 10$, septembre 2002.

${ }^{41}$ Chaze (Jean-François), op. cit., p. 22.
} 
management par objectif). Evoquant leur devoir de performance et leur obligation d'efficacité, les agents que nous avons interrogés se positionnent comme des prestataires de service qui s'appliquent à offrir des services et des produits performants et adaptés. Aujourd'hui, soulignent nos interlocuteurs, les cadres territoriaux rationalisent leurs outils de travail et recourent au quotidien à des tableaux de bord, des suivis journaliers où sont priorisées toutes les actions des agents. «Dans les discussions, il n'est pas rare de parler de portefeuilles d'opérations, de best practice. De plus en plus, on fait appel aux fondamentaux de gestion comme Artémis $7^{42}$ »(Frédéric V.,). «Couramment, explique un directeur de service municipal, on parle ratios, on sait combien coûte un équipement à l'heure, combien coûte notre personnel, il y a de plus en plus d'indicateurs, des plans de financement pluriannuels, des projets d'action » (Dominique).

Une approche économique de leurs services est la volonté de ces dirigeants territoriaux qui entendent transformer l'image de la fonction publique et des professionnels qui la gèrent et la managent. Leur quotidien est une lutte contre le gaspillage, pour une gestion responsable des dépenses publiques et pour le développement de concepts leur permettant d'offrir des prestations de qualité. Leur métier a changé. Ils ne sont plus des techniciens ou des administrateurs occasionnels, ils se voient désormais comme de véritables gestionnaires territoriaux.

\section{Pour conclure}

Ainsi, l'étude de l'organisation territoriale des sports montre que l'autonomie s'est peu à peu réalisée en même temps que s'affirmait une double spécialisation institutionnelle et fonctionnelle. La création de la catégorie A dans la filière sportive a renforcé la stratification et la spécialisation des niveaux de responsabilités entre les agents relevant de trois cadres d'emplois spécifiques. "Les gens en place ont été amenés à compléter leurs connaissances, leurs acquis professionnels, à continuer de se former $\gg$ (Fred). Et cela ne s'est pas fait sans tensions et résistances. Devenus plus «paperassiers» (Fred) et «bureaucrates 》 (Philippe), ils restent toutefois conscients qu'ils doivent s'adapter aux exigences croissantes. Ce sont des fonctionnaires, comme l'a désigné Crozier à propos de l'administration publique d'Etat ${ }^{43}$, à la fois stables par leur statut et les droits qui les protègent (la sécurité de l'emploi notamment) et à la fois sous pression car contraints de mettre en application la politique définie par des élus de plus en plus souvent actifs et jeunes. Ces agents, en quête de reconnaissance au sein d'un service public local plus performant, ont largement contribué à cette évolution gestionnaire. Issus désormais de formations universitaires de haut niveau et validant des concours de plus en plus sélectifs, ils opposent, à une posture jugée trop technicienne de leur métier, une vision de gestionnaires assumant un devoir de performance et de rationalisation de leur mission de service public. Cette réalité est la conséquence de la décentralisation et du transfert massif des compétences qui conduisent les collectivités à adopter une gestion plus rationnelle et soucieuse de performance dans une logique de prestataire de service public. Elle est aussi la traduction de la professionnalisation des métiers territoriaux qui nécessite une adaptation des compétences et des formations aux situations locales, incitant les agents territoriaux à recourir aux outils et aux méthodes de travail faisant appel aux sciences de gestion et de management.

Dans le vaste «mercato ${ }^{44}$ des collectivités territoriales, il ne fait guère de doute que l'approche gestionnaire des personnels territoriaux est de plus en plus recherchée, soulignant

\footnotetext{
${ }^{42}$ Artémis 7 est un logiciel de contrôle et de pilotage des investissements et des opérations s'y référant.

${ }^{43}$ Crozier (Michel), op. cit., p.765.

${ }^{44}$ Dossier Expert, «Collectivités territoriales : Les élections activent le marché des cadres responsables », Journal Le Monde, 22 janvier 2008.
} 
la reconfiguration d'une gouvernance territoriale des sports à laquelle les agents veulent aussi participer. 
Méthodologie d'enquête

Pour mener cette étude, nous nous sommes basés sur un échantillon de 37 agents et cadres de la filière sportive territoriale, construit en réseau et selon le principe de saturation. Nous les avons interrogés sur leurs lieux de travail, dans des lieux publics ou par téléphone. Nous tenons à les remercier d'avoir gentiment accepté de répondre à toutes nos questions.

\begin{tabular}{|c|c|c|c|c|c|c|c|c|}
\hline $\begin{array}{l}\text { Collectivités par } \\
\text { nombre d'habitants }\end{array}$ & $\begin{array}{c}\text { Nom des } \\
\text { Collectivités }\end{array}$ & $\begin{array}{l}\text { Personnes } \\
\text { interviewées }\end{array}$ & Age & $\begin{array}{c}\text { Diplôme } \\
\text { scolaire }\end{array}$ & Diplôme sportif & Niveau sportif & $\begin{array}{l}\text { Cadre d'emploi des personnes } \\
\text { interviewées }\end{array}$ & $\begin{array}{c}\text { Fonctions exercées par les personnes } \\
\text { interviewées }\end{array}$ \\
\hline \multirow{2}{*}{$\begin{array}{l}\text { Commune de moins } \\
\text { de } 5000 \text { habitants }\end{array}$} & Collégien & Christophe J & 42 & $\begin{array}{l}\text { Maîtrise } \\
\text { staps }\end{array}$ & $\begin{array}{l}\text { BE (natation, pour } \\
\text { tous, cyclisme, } \\
\text { VTT) }\end{array}$ & National & $\begin{array}{c}\text { Conseiller territorial des activités } \\
\text { physiques et sportives }\end{array}$ & Directeur du service des sports \\
\hline & Fontenay-Trésigny & Jean & 49 & BEPC & BE natation & Moyen & $\begin{array}{c}\text { Educateur territorial des activités } \\
\text { physiques et sportives }\end{array}$ & $\begin{array}{l}\text { Chef de bassin et maittre nageur } \\
\text { sauveteur }\end{array}$ \\
\hline \multirow{2}{*}{$\begin{array}{l}\text { Commune de } 5000 \text { à } \\
10000 \text { habitants }\end{array}$} & Morez & Philippe P & - & Bac F3 & BE foot & Moyen & $\begin{array}{c}\text { Educateur territorial des activités } \\
\text { physiques et sportives }\end{array}$ & $\begin{array}{c}\text { Enseignant des activités physiques et } \\
\text { sportives }\end{array}$ \\
\hline & Emerainville & Lila & - & Bac pro & BE natation & Moyen & $\begin{array}{c}\text { Educateur territorial des activités } \\
\text { physiques et sportives }\end{array}$ & $\begin{array}{l}\text { Entraîneur, enseignant des activités } \\
\text { physiques et sportives et maître nageur } \\
\text { sauveteur }\end{array}$ \\
\hline $\begin{array}{l}\text { Commune de } 10000 \\
\text { à } 20000 \text { habitants }\end{array}$ & Argentan & Sophie & 23 & Bac S & BE natation & Moyen & $\begin{array}{c}\text { Educateur territorial des activités } \\
\text { physiques et sportives }\end{array}$ & maitre nageur sauveteur \\
\hline \multirow{6}{*}{$\begin{array}{l}\text { Commune de } 20000 \\
\text { à } 25000 \text { habitants }\end{array}$} & $\begin{array}{l}\text { Pavillons-sous- } \\
\text { Bois }\end{array}$ & Loïc & 31 & $\begin{array}{l}\text { Licence } \\
\text { staps }\end{array}$ & $\begin{array}{c}\mathrm{BE} \\
\text { pour tous, } \mathrm{BF}\end{array}$ & National & $\begin{array}{l}\text { Ancien éducateur territorial des } \\
\text { activités physiques et sportives }\end{array}$ & Agent immobilier \\
\hline & Roissy-en-Brie & Emmanuel & 36 & $\begin{array}{l}\text { Licence } \\
\text { économie }\end{array}$ & $\begin{array}{l}\mathrm{BE} \text { natation } \\
\mathrm{BF} \text { triathlon }\end{array}$ & Moyen & $\begin{array}{c}\text { Educateur territorial des activités } \\
\text { physiques et sportives }\end{array}$ & $\begin{array}{l}\text { Entraîneur des activités physiques et } \\
\text { sportives et maître nageur sauveteur }\end{array}$ \\
\hline & Roissy-en-Brie & Arnaud & 26 & $\begin{array}{l}\text { Licence } \\
\text { staps }\end{array}$ & BE natation & Moyen & $\begin{array}{c}\text { Educateur territorial des activités } \\
\text { physiques et sportives }\end{array}$ & maître nageur sauveteur \\
\hline & Ozoir-la-Ferrière & Mathieu & 20 & Bac $\mathrm{S}$ & $\begin{array}{l}\text { BE natation } \\
\mathrm{BF} \text { triathlon }\end{array}$ & Moyen & $\begin{array}{c}\text { Educateur territorial des activités } \\
\text { physiques et sportives }\end{array}$ & Maître nageur sauveteur \\
\hline & $\begin{array}{l}\text { SAN du Val } \\
\text { d'Europe }\end{array}$ & Franck & 45 & $\begin{array}{l}\text { Licence } \\
\text { staps }\end{array}$ & $\begin{array}{l}\text { BE plongée, pour } \\
\text { tous }\end{array}$ & National & $\begin{array}{c}\text { Conseiller territorial des activités } \\
\text { physiques et sportives }\end{array}$ & Directeur du service des sports \\
\hline & Torcy & Gérard & 63 & $1^{\text {ere }} \mathrm{S}$ & BE natation & Moyen & $\begin{array}{c}\text { Educateur territorial des activités } \\
\text { physiques et sportives }\end{array}$ & $\begin{array}{c}\text { Chef de bassin et maittre nageur } \\
\text { sauveteur }\end{array}$ \\
\hline \multirow{2}{*}{$\begin{array}{l}\text { Commune de } 25000 \\
\text { à } 35000 \text { habitants }\end{array}$} & Gagny & Patrick & - & $\mathrm{Bac}$ & BE natation & Moyen & $\begin{array}{c}\text { Educateur territorial des activités } \\
\text { physiques et sportives }\end{array}$ & $\begin{array}{l}\text { Responsable des ETAPS et du club de } \\
\text { gymnastique }\end{array}$ \\
\hline & $\begin{array}{l}\text { Pontault-Combault } \\
\end{array}$ & Philippe & 42 & Bac & BE Handball & International & Conseiller territorial des activités & Responsable du service des sports \\
\hline
\end{tabular}




\begin{tabular}{|c|c|c|c|c|c|c|c|c|}
\hline & & & & & & & physiques et sportives & \\
\hline & Pontault-Combault & Saïd & 22 & Bac $S$ & BE handball & International & $\begin{array}{l}\text { Educateur territorial des activités } \\
\text { physiques et sportives }\end{array}$ & $\begin{array}{l}\text { Entraîneur, enseignant des activités } \\
\text { physiques et sportives }\end{array}$ \\
\hline \multirow{3}{*}{$\begin{array}{l}\text { Commune de } 35000 \\
\text { à } 55000 \text { habitants }\end{array}$} & Blanc-Mesnil & Richard & 36 & Bac pro & BE natation & Moyen & $\begin{array}{l}\text { Educateur territorial des activités } \\
\text { physiques et sportives }\end{array}$ & maître nageur sauveteur \\
\hline & Meaux & Henri & 30 & BEP & BE natation & Moyen & $\begin{array}{l}\text { Educateur territorial des activités } \\
\text { physiques et sportives }\end{array}$ & $\begin{array}{l}\text { Entraîneur, enseignant des activités } \\
\text { physiques et sportives }\end{array}$ \\
\hline & Villejuif & Bruno & 27 & $\begin{array}{c}\text { Bac } \\
\text { médico- } \\
\text { social }\end{array}$ & BE pour tous & National & $\begin{array}{l}\text { Educateur sportif des activités } \\
\text { physiques et sportives pour tous }\end{array}$ & $\begin{array}{c}\text { Enseignant des activités physiques et } \\
\text { sportives }\end{array}$ \\
\hline \multirow{4}{*}{$\begin{array}{l}\text { Commune de } 70000 \\
\text { à } 100000 \text { habitants }\end{array}$} & Aubervilliers & Fred & 58 & $\mathrm{Bac}$ & - & Moyen & $\begin{array}{l}\text { Ancien conseiller territorial des } \\
\text { activités physiques et sportives }\end{array}$ & $\begin{array}{l}\text { Directeur du service des sports et de la } \\
\text { jeunesse }\end{array}$ \\
\hline & Créteil & Dominique & & & & & $\begin{array}{c}\text { Conseiller territorial des activités } \\
\text { physiques et sportives }\end{array}$ & Directeur de la base nautique \\
\hline & Créteil & Guéric & 36 & Bac & BE Handball & International & Contractuel & Chargé de mission \\
\hline & Dunkerque & Nicolas & & & & & Attaché territorial & Responsable de la communication \\
\hline \multirow{9}{*}{$\begin{array}{l}\text { Commune et } \\
\text { regroupement de } \\
\text { communes de } \\
100000 \text { à } 200000 \\
\text { habitants }\end{array}$} & Besançon & Nathalie & & $\begin{array}{l}\text { DESS } \\
\text { staps }\end{array}$ & BF kayac, ski & Moyen & $\begin{array}{l}\text { Conseiller territorial des activités } \\
\text { physiques et sportives }\end{array}$ & Directrice adjointe des sports \\
\hline & Besançon & Patrick P & & & & & $\begin{array}{l}\text { Conseiller territorial des activités } \\
\text { physiques et sportives }\end{array}$ & $\begin{array}{l}\text { Responsable des installations nautiques } \\
\text { et de la patinoire }\end{array}$ \\
\hline & Besançon & Frédéric & 48 & $\begin{array}{l}\text { Master } \\
\text { gestion }\end{array}$ & - & $\begin{array}{c}\text { International } \\
\text { water-polo }\end{array}$ & $\begin{array}{c}\text { Conseiller territorial des activités } \\
\text { physiques et sportives }\end{array}$ & $\begin{array}{l}\text { Responsable des secteurs de l'hygiène } \\
\text { et de la sécurité }\end{array}$ \\
\hline & Besançon & Gérard C & 40 & Bac D & BE handball & National & $\begin{array}{l}\text { Conseiller territorial des activités } \\
\text { physiques et sportives }\end{array}$ & Responsable des animateurs sportifs \\
\hline & Besançon & Farid & - & Bac & BE natation & Moyen & $\begin{array}{c}\text { Educateur territorial des activités } \\
\text { physiques et sportives }\end{array}$ & $\begin{array}{l}\text { maître nageur sauveteur et } \\
\text { enseignement des activités aquatiques }\end{array}$ \\
\hline & Besançon & Henri & & & & & $\begin{array}{c}\text { Conseiller territorial des activités } \\
\text { physiques et sportives }\end{array}$ & Chef de projets \\
\hline & Besançon & Jean & & & & & Agent technique & $\begin{array}{c}\text { Responsable des gymnases et des } \\
\text { terrains }\end{array}$ \\
\hline & Besançon & Daniel & & & & & $\begin{array}{c}\text { Conseiller territorial des activités } \\
\text { physiques et sportives }\end{array}$ & Responsable des manifestations \\
\hline & $\begin{array}{c}\text { CA de } \\
\text { Montbéliard }\end{array}$ & Michel & & BEP & $\begin{array}{l}\text { BE kayak, pour } \\
\text { tous }\end{array}$ & Moyen & $\begin{array}{c}\text { Conseiller territorial des activités } \\
\text { physiques et sportives }\end{array}$ & Responsable des activités sportives \\
\hline
\end{tabular}




\begin{tabular}{|c|c|c|c|c|c|c|c|c|}
\hline \multirow{2}{*}{$\begin{array}{l}\text { Commune de } \\
200000 \text { à } 400000 \\
\text { habitants }\end{array}$} & CU de Dunkerque & Frédéric V & & & & & Ingénieur territorial & $\begin{array}{l}\text { Responsable communication et } \\
\text { responsable de formation au CNFPT }\end{array}$ \\
\hline & SAN de Sénart & Christophe N & 38 & $\begin{array}{c}\text { DESS } \\
\text { Droit éco. }\end{array}$ & BE Handball & Moyen & $\begin{array}{l}\text { Conseiller territorial des activités } \\
\text { physiques et sportives }\end{array}$ & Directeur du service des sports \\
\hline \multirow{2}{*}{$\begin{array}{l}\text { Département de } \\
1300000 \text { habitants }\end{array}$} & $\begin{array}{l}\text { Conseil général de } \\
\text { Seine-et-Marne }\end{array}$ & Jean-Luc & 45 & $\begin{array}{l}\text { Licence } \\
\text { staps }\end{array}$ & - & Moyen & $\begin{array}{l}\text { Conseiller territorial des activités } \\
\text { physiques et sportives }\end{array}$ & Responsable du service des sports \\
\hline & $\begin{array}{l}\text { Conseil général de } \\
\text { Seine-et-Marne }\end{array}$ & Hadi & 35 & $\begin{array}{l}\text { Master } \\
\text { staps }\end{array}$ & $\begin{array}{l}\text { BE pour tous, } \mathrm{BF} \\
\text { voile kayak roller }\end{array}$ & Moyen & $\begin{array}{c}\text { Educateur territorial des activités } \\
\text { physiques et sportives }\end{array}$ & $\begin{array}{l}\text { Responsable des relations avec les } \\
\text { comités }\end{array}$ \\
\hline \multirow{2}{*}{$\begin{array}{l}\text { Ville de } 2100000 \\
\text { habitants }\end{array}$} & Paris & Yohan & 25 & Bac S & $\begin{array}{l}\text { BE Handball, } \\
\text { pour tous }\end{array}$ & Régional & $\begin{array}{l}\text { Professeur d'Education physique et } \\
\text { sportive }\end{array}$ & $\begin{array}{c}\text { Entraîneur, enseignant des activités } \\
\text { physiques et sportives }\end{array}$ \\
\hline & Paris & Odile & 45 & - & BE Handball & International & $\begin{array}{l}\text { Professeur d'éducation physique et } \\
\text { sportive }\end{array}$ & $\begin{array}{c}\text { Enseignant des activités physiques et } \\
\text { sportives }\end{array}$ \\
\hline CNFPT & Paris & Jean-Louis & & Licence & & Moyen & $\begin{array}{c}\text { Conseiller territorial des activités } \\
\text { physiques et sportives }\end{array}$ & $\begin{array}{l}\text { Responsable de formation des CTAPS } \\
\text { (sport, développement des territoires et } \\
\text { environnement) }\end{array}$ \\
\hline
\end{tabular}

